\title{
Protective Effect of Dimethyl Fumarate on Memory Impairment After Cerebral Ischemia-Reperfusion Injury in Rats
}

\author{
Sasan Dastaran ${ }^{1}$, Masoumeh Sabetkasaei ${ }^{1,{ }^{*}}$, Siavash Parvardeh ${ }^{1}$, Shiva Ghafghazi ${ }^{1}$ and Ali Pouramini ${ }^{1}$ \\ ${ }^{1}$ Department of Pharmacology, School of Medicine, Shahid Beheshti University of Medical Sciences, Tehran, Iran \\ "Corresponding author: Department of Pharmacology, School of Medicine, 5th Floor, Bldg. No.1, Shahid Beheshti University of Medical Sciences, Daneshjoo Blvd, Velenjak, \\ Postal Code: 1983963113, Tehran, Iran. Tel/Fax:+98-2122439969, Email: kasai@sbmu.ac.ir
}

Received 2018 April 10; Revised 2019 May 09; Accepted 2018 May 15.

\begin{abstract}
Background: Cerebral ischemia is an important cause of morbidity and mortality worldwide. Dimethyl fumarate (DMF) is indicated for the treatment of patients with relapsing forms of Multiple Sclerosis and psoriasis.

objectives: In this study, the effect of DMF on memory and learning impairments and nitrosative stress after cerebral ischemia in rats, was evaluated.

Methods: Cerebral ischemia was induced via common carotid artery occlusion (CCAO). Rats were randomly divided into three groups $(\mathrm{N}=5)$. Group I included Sham-operated animals who underwent surgery without arterial occlusions, group II (control ischemic) underwent surgery to induce transient global cerebral ischemia for $20 \mathrm{~min}$ and received $0.2-0.25 \mathrm{~mL}$ of distilled water, twice a day by oral gavage, group III included rats which underwent cerebral ischemia and then received DMF (15 mg/kg, twice daily, for 1 week) by oral gavage. Morris water maze test was used to assess spatial memory. Nitric oxide (NO) level in the hippocampus was measured using Griess test.

Results: Treatment of rats with DMF (15 mg/kg, orally, twice daily for 7 days) resulted in a significant decrease in escape latency during training trials. Besides, the time spent in the target quadrant and the number of crossings over the platform area were significantly increased in the DMF-treated rats which were accompanied by a decrease in the proximity to the platform in the probe trial. Furthermore, the results of the Griess assay indicated a significant reduction in the NO levels in the hippocampus of DMFtreated rats.
\end{abstract}

Conclusions: Overall, our findings indicate that DMF improves memory impairment induced by cerebral ischemia/reperfusion injury in rats through the suppression of nitrosative stress in the hippocampus.

Keywords: Dimethyl Fumarate, Cerebral Ischemia, Memory Deficit, Nitric Oxide

\section{Background}

Ischemic brain injury is one of the most important causes of disability and mortality in adults worldwide that results from cerebral circulatory arrest $(1,2)$. Transient global cerebral ischemia (TGCI) can cause cognitive, behavioral, learning and memory impairments $(2,3)$. Characterized mechanisms of post-TGCI neuronal damage consist of diminished blood flow to the brain, mitochondrial damage, free radical development, destruction of membrane, injury to the blood vessels, prevention of protein synthesis, inflammation, brain edema formation and finally delayed neuronal death of the CA1 zone of the hippocampus (4).

Ischemia/reperfusion injury via lipid peroxidation leads to increased reactive oxygen species (ROS) and therefore causes mitochondrial dysfunction, interruption of the membrane, alterations in permeability and ultimately cell death (5).

Nitric oxide (NO) is another factor involved in injuries after cerebral ischemia/reperfusion, that is generated by various sources such as neurons and glia (6), and can cause increased levels of ROS, consumption of adenosine triphosphate (ATP) and neuronal death (7).

There have been numerous researches concentrating on several neuroprotective agents for preventing the secondary neuronal damage following ischemia/reperfusion. Despite all efforts, at present we do not have an approved pharmacological therapy for preventing neuronal injuries following cerebral ischemia/reperfusion.

Dimethyl fumarate (DMF) is the methyl ester of fumaric acid with confirmed indications in patients with chronic plaque psoriasis, a dermatological sickness with immune system dysfunction and relapsing-remitting multiple sclerosis (8). 


\section{Objectives}

The aim of this novel study was to investigate the effects of DMF on memory impairment and NO levels following cerebral ischemia in rats.

\section{Methods}

\subsection{Chemicals and Drugs}

Chemicals were bought from Sigma-Aldrich Chemical Co. As per the safety sheet issued by Sigma-Aldrich, Oral LD50 in rat is $2240 \mathrm{mg} / \mathrm{kg}$.

DMF was dissolved in distilled water and was used at a dosage of $15 \mathrm{mg} / \mathrm{kg}$ body weight based on previous researches (9), twice daily (every 12 hours) via oral gavage for a period of 7 days after ischemia.

Chemical compounds studied in this article: DMF(PubChem CID: 637568).

\subsection{Animal}

The male Wistar rats, 200 - 250 g, were obtained from the animal house of Shahid Beheshti University of Medical Sciences, Tehran, Iran, and maintained under steady conditions (12 h/12 h light/dark cycle), at a controlled temperature of $23 \pm 2^{\circ} \mathrm{C}$. Standard pellet diet and tap water were available.

\subsection{Study Design}

Rats were randomly allocated to three groups $(\mathrm{N}=$ 5). Group I included Sham-operated animals undergoing surgery without arterial occlusions, group II (control ischemic) received $0.2-0.25 \mathrm{~mL}$ of distilled water, twice a day by oral gavage, group III included rats undergoing surgery and cerebral ischemia and then received DMF $(15 \mathrm{mg} / \mathrm{kg}$, twice daily, for 1 week) by oral gavage.

\subsection{Surgical Procedure}

Before surgery procedure, rats were anesthetized with intraperitoneal administration of xylazine $(5 \mathrm{mg} / \mathrm{kg})$ and ketamine hydrochloride (50 mg/kg) (10).

Transient global cerebral ischemia was performed by common carotid arteries occlusion (CCAO) for $20 \mathrm{~min}$, using the microvascular clamps. Subsequently, both clamps were removed and both arteries inspected for immediate reperfusion (11).

\subsection{Morris Water Maze}

The water maze is a circular tank $120 \mathrm{~cm}$ in diameter and $60 \mathrm{~cm}$ in height. The tank was filled with water $(23 \pm$ $2^{\circ} \mathrm{C}$ ) to a depth of $40 \mathrm{~cm}$. The maze was located in a room containing extra-maze cues (posters). The maze was divided geographically into four quadrants (northeast (NE), northwest (NW), southeast (SE), southwest (SW)) and starting positions (north $(\mathrm{N})$, south $(\mathrm{S})$, east $(\mathrm{E})$, west $(\mathrm{W})$ ) that were equally spaced around the perimeter of the pool. A hidden circular platform (diameter: $12 \mathrm{~cm}$ ) was installed in the center of the NW quadrant, $1.5 \mathrm{~cm}$ below the surface of the water. A video camera was mounted directly above the maze to record the rats' swim paths. A computer tracking system was used to measure the escape latency, time spent, traveled distance, the number of crossings, average proximity and average swimming speeds in the target quad$\operatorname{rant}(12,13)$.

\subsection{Measurement of Nitrite in the Hippocampus}

The hippocampus was quickly removed following decapitation and homogenized $(5: 1 \mathrm{v} / \mathrm{w})$ in ice-cold $0.1 \mathrm{M}$ phosphate buffer, $\mathrm{pH}$ 7.4. The nitrite concentration in the hippocampal tissue was utilized as a measure of NO generation. Which was determined by measuring nitrite accumulation in the medium by Griess reagent (1\% sulfanilamide and 0.1\% N-(1-naphthyl)-ethylenediamine dihydrochloride in $5 \% \mathrm{H}_{3} \mathrm{PO}_{4}$ ). Then, $100 \mu \mathrm{L}$ of supernatant and $100 \mu \mathrm{L}$ Griess reagent were mixed and incubated for $5 \mathrm{~min}$. The absorption was estimated in a microplate reader at 540 $\mathrm{nm}$ (14).

\subsection{Statistical Analysis}

All the results are presented as mean \pm SEM. Statistical analyses were carried out using PRISM 6.0. Analysis of variance test was applied to determine statistical significance. A P value $<0.05$ was regarded as statistically significant.

\section{Results}

4.1. Effect of DMF on Spatial Memory in the Morris Water Maze Test

Results analysis of the Morris water maze during training trials (first 3 days), escape latency (time to find the hidden platform) in DMF (15 mg/kg) and sham groups were significantly decreased $(\mathrm{P}<0.05)$ in comparison with control group. eEscape latency on the third day of the training trial was the lowest (Figure 1). Additionally in probe trial, time spent and traveled distance in goal quadrant during the experiment, in DMF and sham groups were significantly increased $(\mathrm{P}<0.05)$ in comparison with control group (Figures 2 and 3, respectively) Furthermore, in the probe trial the number of crossings on the platform's 
place was significantly enhanced $(\mathrm{P}<0.05)$ in DMF and sham groups in comparison with control group (Figure 4). Moreover, in the probe trial, mean proximity to platform in $\operatorname{DMF}(15 \mathrm{mg} / \mathrm{kg})$ was significantly different compared to the control group $(\mathrm{P}<0.05)$ (Figure 5) but average swimming speed during the probe trial in Morris water maze test, did not show a significant difference between groups (Figure $6)$.

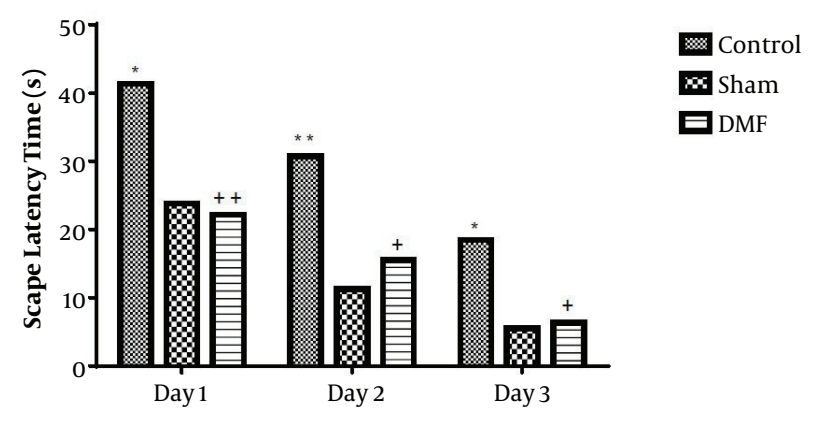

$\overline{\text { Figure 1. Escape latency time (mean } \pm \text { SEM) during training days, to reach a hidden }}$ platform in rats treated by DMF $(15 \mathrm{mg} / \mathrm{kg}$, orally, twice daily, for 1 week $)$ in the Morris water maze test. By Two-way ANOVA, ${ }^{*} \mathrm{P}<0.05,{ }^{* *} \mathrm{P}<0.01$, significantly different compared to the sham, ${ }^{+} \mathrm{P}<0.05,{ }^{++} \mathrm{P}<0.01$, compared with the control group.

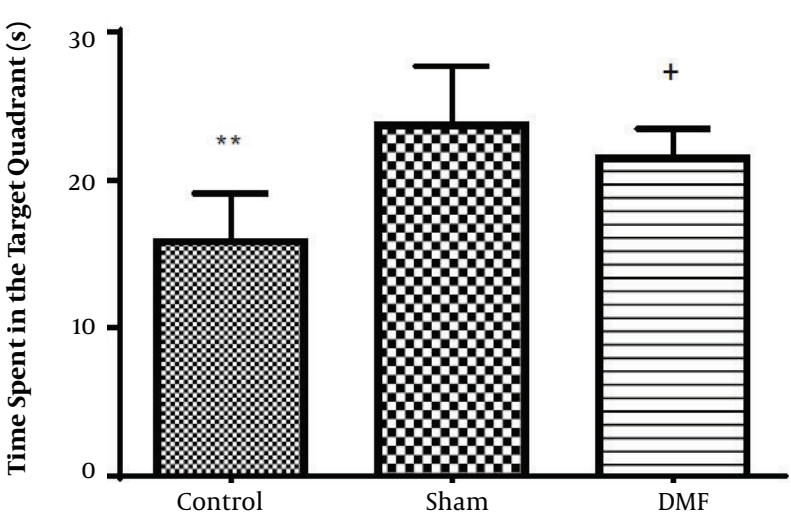

Figure 2. Time spent (mean \pm SEM) in the goal quadrant during probe trial, by rats treated with DMF (15 mg/kg, orally, twice daily, for 1 week) in the Morris water maze test. ${ }^{* *} \mathrm{P}<0.01$, significantly different compared to the sham, ${ }^{+} \mathrm{P}<0.05$ compared with the control group.

\subsection{Effect of DMF on NO Levels}

According to the one-way ANOVA results of this study, nitric oxide level of the hippocampus of control group was increased compared to the sham group. But DMF (15 $\mathrm{mg} / \mathrm{kg}$, twice daily, for 1 week) prevented the rise of the levels of $\mathrm{NO}[\mathrm{F}(2,12)=11.18, \mathrm{P}<0.01]$ in the hippocampus of rats subjected to ischemia/reperfusion injury (Figure 7).

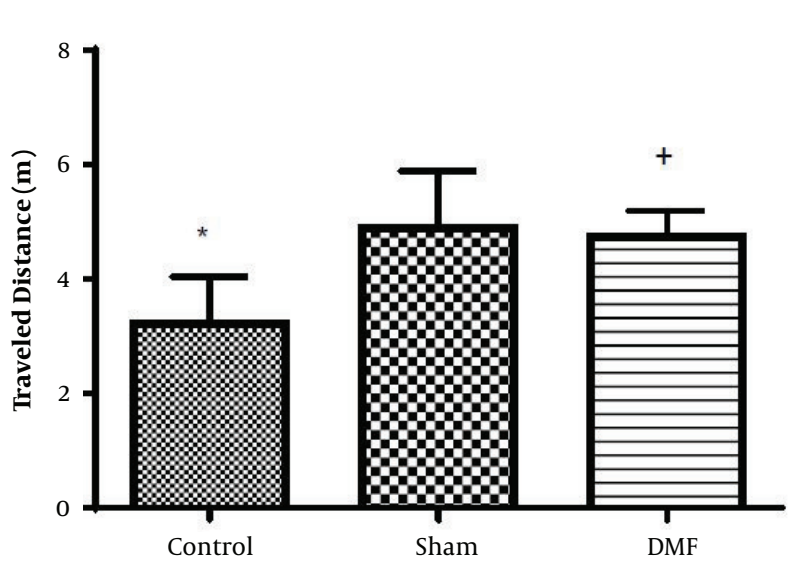

Figure 3. Traveled distance (mean \pm SEM) in the goal quadrant during probe trial by rats treated with DMF (15 mg/kg, orally, twice daily, for 1 week) in the Morris water maze test. ${ }^{*} \mathrm{P}<0.05$ : significantly different compared to the sham, ${ }^{+} \mathrm{P}<0.05 \mathrm{com}$ pared with the control group.

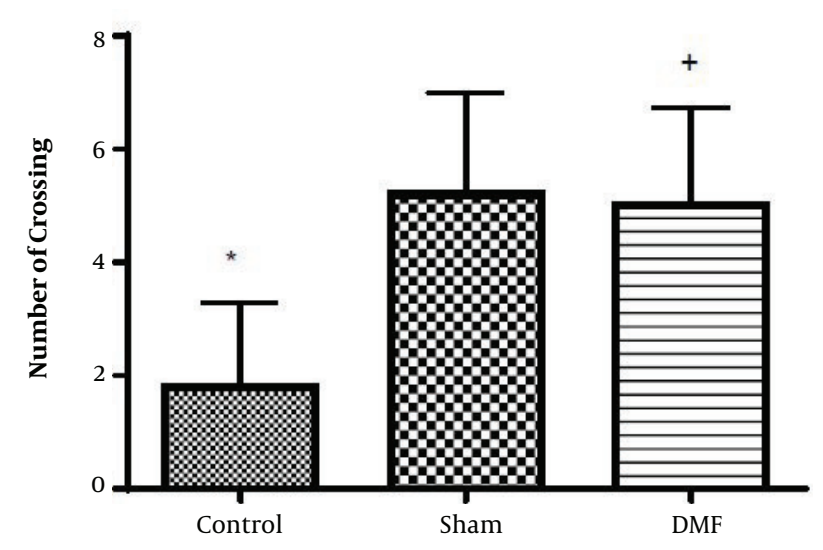

Figure 4. Number of crossing (mean \pm SEM) in the goal quadrant during probe trial, by rats treated with DMF ( $15 \mathrm{mg} / \mathrm{kg}$, orally, twice daily, for 1 week) in the Morris water maze test. ${ }^{*} \mathrm{P}<0.05$, compared with the sham group; ${ }^{+} \mathrm{P}<0.05$, compared with the control group.

\section{Discussion}

Our research proposed the use of DMF for the management and prevention of memory impairments caused by cerebral ischemia/reperfusion. In addition, we investigated the underlying mechanism of DMF with a focus on nitrosative stress. Learning and memory impairments represent the pathogenic advancement of the damage induced by cerebral ischemia/reperfusion. Such damages could lead to disabilities and restriction in social activities $(3,15)$. In our study, we have seen a progress in learning behaviors in rats treated with DMF compared to control group, 7 days after the induction of ischemia by Morris water maze test. 


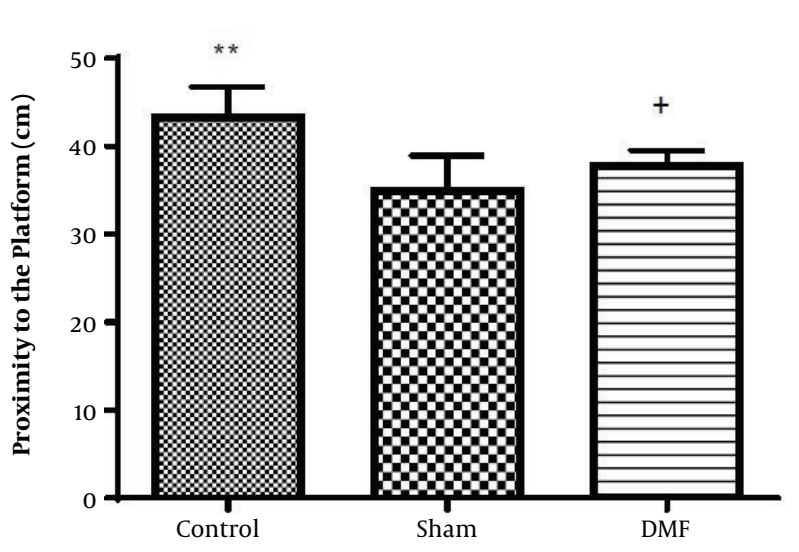

Figure 5. Proximity (mean \pm SEM) to the platform during probe trial in rats treated by DMF ( $15 \mathrm{mg} / \mathrm{kg}$, orally, twice daily, for 1 week) in the Morris water maze test. ${ }^{* *} \mathrm{P}<$ 0.05 compared with the sham group; ${ }^{+} \mathrm{P}<0.05$ compared with the control group.

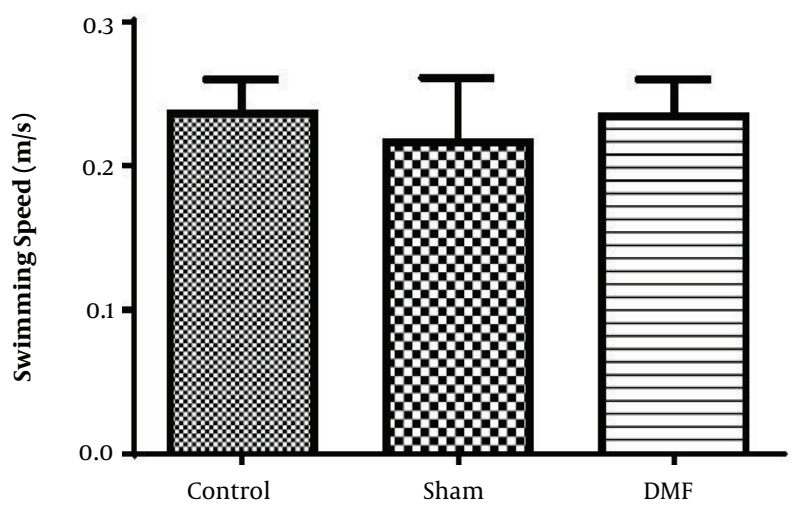

Figure 6. Swim speed (mean \pm SEM) during the probe trial in rats treated by DMF $(15 \mathrm{mg} / \mathrm{kg}$, orally, twice daily, for 1 week) in the Morris water maze test. No significant difference was observed between groups on the average speeds of the rats.

Previously many studies have focused to nominate a pharmacological intervention to attenuate cerebral ischemia injury and subsequent memory impairments and to address that goal, several compounds such as ROS scavengers, glutamate receptor blockers, calcium channel inhibitors were applied (16), but none of them lead to an approved medication for the discussed indication, although experimental studies on numerous neuroprotective and anti-inflammatory agents are still ongoing.

The results obtained from Morris water maze, showed that the rats treated with oral DMF, improved spatial memory, represented by reduction in escape latency (time to find the platform) and proximity of rats to the platform's location as compared to the ischemia group. Also, DMF significantly enhanced time spent in the target quadrant, traveled distance and number of crossings in the goal

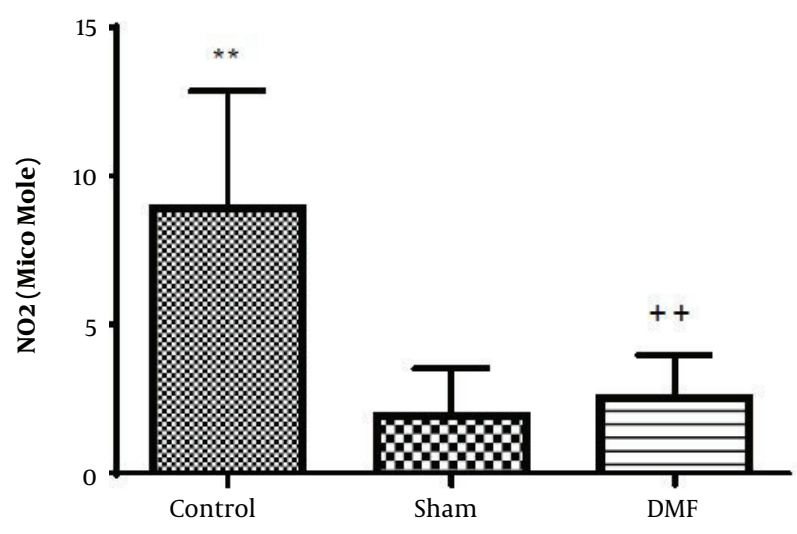

Figure 7. Effect of treatment with DMF (15 mg/kg, orally, twice daily, for 1 week) on the concentration of Nitric Oxide in the hippocampus of rats subjected to transient cerebral ischemia/reperfusion. Data are reported as mean $\pm S E M ;(n=5 /$ group $) .{ }^{* *} \mathrm{P}$ $<0.01$ compared with sham group; ${ }^{++} \mathrm{P}<0.01$ compared with the control group

quadrant in comparison with the ischemia group. No significant difference was found in swimming speed between the ischemia, DMF and Sham group in the probe trial, on the 4th day of Morris water maze test. Hence the concern that motor impairment upon surgery could affect our data, can be excluded. From the other side as a result, since the mean speeds of the rats in trial day were not differ significantly between groups, the effects of DMF on spatial navigation do not appear to be relevant to improvement in motor ability.

Also in experiment comparing of escape latency in each day, we observed that the escape latency on the third day in the DMT-treated rats was significantly shorter in comparison with the control group. The latter finding also supports the other data that DMF might be able to improve learning functionality of ischemic rats.

Enhanced levels of NO have been shown as another factor involved in the injuries associated with cerebral ischemia/reperfusion and may worsen the primary damages to neurons $(17,18)$. Furthermore, NO reacts with superoxide anion radical $\left(\mathrm{O}_{2}{ }^{-}\right)$to produce per-oxynitrite, a very reactive molecule in lipid oxidation, proteins and DNA; that again leads to further increased inflammation, microglial cells destruction and CNS injury $(19,20)$.

One of the major findings of our research, is that DMF can prevent increased levels of NO after cerebral ischemia/reperfusion in rats hence, prevention of the boost of NO, can be considered as another beneficial effect of DMF in complications resulting from cerebral ischemia. Some other studies have shown that prevention of increased NO levels by various compounds including DMF can be employed to restrain the progression of neurodegenerative diseases (21-23). 


\subsection{Conclusions}

Our results demonstrate that DMF improves cerebral ischemia-induced learning and memory impairment, and the promising memory-improvement effects of DMF may be mediated through reduction of the levels of NO in the hippocampus.

\section{Footnotes}

Authors' Contribution: Sasan Dastaran and Masoumeh Sabetkasaei carried out the experiment. Sasan Dastaran wrote the manuscript with support from Siavash Parvardeh and Masoumeh Sabetkasaei. Ali Pouramini and Shiva Ghafghazi helped supervise the project and animal procedures.

Conflict of Interests: The authors declare that they have no conflict of interest.

Ethical Approval: All applicable institutional and international guidelines for the care and use of animals were followed.

Funding/Support: This study was funded by Shahid Beheshti University of Medical Sciences, School of Medicine.

\section{References}

1. Nikonenko AG, Radenovic L, Andjus PR, Skibo GG. Structural features of ischemic damage in the hippocampus. Anat Rec (Hoboken). 2009;292(12):1914-21. doi:10.1002/ar.20969. [PubMed: 19943345].

2. Neumar RW. Molecular mechanisms of ischemic neuronal injury. Ann Emerg Med. 2000;36(5):483-506. doi: 10.1067/mem.2000.110995. [PubMed: 11054204].

3. Vaughan L, Bushnell C, Bell CL, Espeland MA. Global cognitive function before, surrounding, and after ischemic stroke: The role of risk and protective factors varies with time among ischemic stroke survivors. Neuropsychol Dev Cogn B Aging Neuropsychol Cogn. 2016;23(1):117-31. doi: 10.1080/13825585.2015.1058323. [PubMed: 26073439]. [PubMed Central: PMC4916645].

4. Ankarcrona M, Dypbukt JM, Bonfoco E, Zhivotovsky B, Orrenius S, Lipton SA, et al. Glutamate-induced neuronal death: A succession of necrosis or apoptosis depending on mitochondrial function. Neuron. 1995;15(4):961-73. doi: 10.1016/0896-6273(95)90186-8.

5. Nigam $S$, Schewe T. Phospholipase $A(2) s$ and lipid peroxidation. Biochim Biophys Acta. 2000;1488(1-2):167-81. doi: 10.1016/s13881981(00)00119-0. [PubMed: 11080686].

6. Dawson TM, Dawson VL. Review: Nitric oxide: Actions and pathological roles. Neurosci. 2016;1(1):7-18. doi: 10.1177/107385849500100103.

7. Goto S, Xue R, Sugo N, Sawada M, Blizzard KK, Poitras MF, et al. Poly(ADP-ribose) polymerase impairs early and long-term experimental stroke recovery. Stroke. 2002;33(4):1101-6. doi: 10.1161/01.str.0000014203.65693.1e. [PubMed: 11935067].

8. Gold R, Kappos L, Arnold DL, Bar-Or A, Giovannoni G, Selmaj K, et al. Placebo-controlled phase 3 study of oral BG-12 for relapsing multiple sclerosis. N Engl J Med. 2012;367(12):1098-107. doi: 10.1056/NEJMoa1114287. [PubMed: 22992073].
9. Kunze R, Urrutia A, Hoffmann A, Liu H, Helluy X, Pham M, et al. Dimethyl fumarate attenuates cerebral edema formation by protecting the blood-brain barrier integrity. Exp Neurol. 2015;266:99-111. doi: 10.1016/j.expneurol.2015.02.022. [PubMed: 25725349].

10. [No authors listed]. Novel techniques for the study of drugs and behaviour. Behav Pharmacol. 2017;28(8):595-6. doi: 10.1097/FBP.0000000000000367. [PubMed: 29120947].

11. Wu CP, Cheung G, Rakhshani N, Parvardeh S, Asl MN, Huang HL, et al. Ca3 neuronal activities of dorsal and ventral hippocampus are differentially altered in rats after prolonged post-ischemic survival. Neuroscience. 2005;130(2):527-39. doi: 10.1016/j.neuroscience.2004.09.041. [PubMed: 15664709].

12. Hosseinzadeh H, Asl MN, Parvardeh S, Tagi Mansouri SM. The effects of carbenoxolone on spatial learning in the Morris water maze task in rats. Med Sci Monit. 2005;11(3):BR88-94. [PubMed: 15735560].

13. Moghimi M, Parvardeh S, Zanjani TM, Ghafghazi S. Protective effect of alpha-terpineol against impairment of hippocampal synaptic plasticity and spatial memory following transient cerebral ischemia in rats. Iran J Basic Med Sci. 2016;19(9):960-9. [PubMed: 27803783]. [PubMed Central: PMC5080426].

14. Wilms H, Sievers J, Rickert U, Rostami-Yazdi M, Mrowietz U, Lucius R. Dimethylfumarate inhibits microglial and astrocytic inflammation by suppressing the synthesis of nitric oxide, IL-1beta, TNF-alpha and IL-6 in an in-vitro model of brain inflammation. J Neuroinflammation. 2010;7:30. doi: 10.1186/1742-2094-7-30. [PubMed: 20482831]. [PubMed Central: PMC2880998].

15. Muir KW, Tyrrell P, Sattar N, Warburton E. Inflammation and ischaemic stroke. Curr Opin Neurol. 2007;20(3):334-42. doi: 10.1097/WCO.0b013e32813ba151. [PubMed: 17495630].

16. Ozbal S, Erbil G, Kocdor H, Tugyan K, Pekcetin C, Ozogul C. The effects of selenium against cerebral ischemia-reperfusion injury in rats. Neurosci Lett. 2008;438(3):265-9. doi: 10.1016/j.neulet.2008.03.091. [PubMed: 18490106].

17. Harukuni I, Bhardwaj A. Mechanisms of brain injury after global cerebral ischemia. Neurol Clin. 2006;24(1):1-21. doi: 10.1016/j.ncl.2005.10.004. [PubMed: 16443127].

18. McGeer PL, McGeer EG. The inflammatory response system of brain: Implications for therapy of Alzheimer and other neurodegenerative diseases. Brain Res Brain Res Rev. 1995;21(2):195-218. doi: 10.1016/01650173(95)00011-9. [PubMed: 8866675].

19. Basso AS, Frenkel D, Quintana FJ, Costa-Pinto FA, Petrovic-Stojkovic S, Puckett L, et al. Reversal of axonal loss and disability in a mouse model of progressive multiple sclerosis.JClin Invest. 2008;118(4):1532-43. doi: 10.1172/JCI33464. [PubMed: 18340379]. [PubMed Central: PMC2267014].

20. Hill KE, Zollinger LV, Watt HE, Carlson NG, Rose JW. Inducible nitric oxide synthase in chronic active multiple sclerosis plaques: Distribution, cellular expression and association with myelin damage. J Neuroimmunol. 2004;151(1-2):171-9. doi: 10.1016/j.jneuroim.2004.02.005. [PubMed: 15145615].

21. Goyagi T, Toung TJ, Kirsch JR, Traystman RJ, Koehler RC, Hurn PD, et al. Neuroprotective kappa-opioid receptor agonist BRL 52537 attenuates ischemia-evoked nitric oxide production in vivo in rats. Stroke. 2003;34(6):1533-8. doi: 10.1161/01.STR.0000072512.30658.E7. [PubMed: 12738895].

22. Li ST, Pan J, Hua XM, Liu H, Shen S, Liu JF, et al. Endothelial nitric oxide synthase protects neurons against ischemic injury through regulation of brain-derived neurotrophic factor expression. CNS Neurosci Ther. 2014;20(2):154-64. doi: 10.1111/cns.12182. [PubMed: 24397751]. [PubMed Central: PMC6493204].

23. Zeynalov E, Nemoto M, Hurn PD, Koehler RC, Bhardwaj A. Neuroprotective effect of selective kappa opioid receptor agonist is gender specific and linked to reduced neuronal nitric oxide. J Cereb Blood Flow Metab. 2006;26(3):414-20. doi: 10.1038/sj.jcbfm.9600196. [PubMed: 16049424]. 